\title{
Cortical $\beta$-amyloid burden, neuropsychiatric symptoms, and cognitive status: the Mayo Clinic Study of Aging
}

Janina Krell-Roesch ${ }^{1,2}$, Maria Vassilaki ${ }^{3}$, Michelle M. Mielke ${ }^{3,4}$, Walter K. Kremers ${ }^{3}$, Val J. Lowe ${ }^{5}$, Prashanthi Vemuri, Mary M. Machulda ${ }^{6}$, Teresa J. Christianson ${ }^{3}$, Jeremy A. Syrjanen ${ }^{3}$, Gorazd B. Stokin ${ }^{7}$, Lesley M. Butler ${ }^{8}$, Martin Traber ${ }^{8}$, Clifford R. Jack Jr., David S. Knopman ${ }^{4}$, Rosebud O. Roberts ${ }^{3,4}$, Ronald C. Petersen ${ }^{3,4}$ and Yonas E. Geda ${ }^{13,9,10}$

\begin{abstract}
Neuropsychiatric symptoms (NPS) are a risk factor for cognitive impairment and are associated with cortical $\beta$-amyloid (Aß) deposition. We conducted a cross-sectional study derived from the ongoing population-based Mayo Clinic Study of Aging to examine the frequency of NPS among cognitively unimpaired (CU) and mild cognitive impairment (MCI) participants who either have normal $(A-)$ or abnormal $(A+) A \beta$ deposition. We also investigated whether combined presence of $\mathrm{MCl}$ and amyloid positivity $(\mathrm{MCl} / \mathrm{A}+)$ is associated with greater odds of having NPS as compared to CU/A - (defined as reference group). Participants were $1627 \mathrm{CU}$ and MCI individuals aged $\geq 50$ years (54\% males; median age 73 years). All participants underwent NPS assessment (Neuropsychiatric Inventory Questionnaire (NPI-Q); Beck Depression Inventory II (BDI-II); Beck Anxiety Inventory (BAI)) and ${ }^{11} \mathrm{C}$-PiB-PET. Participants with an SUVR $>1.42$ were classified as A+. We conducted multivariable logistic regression analyses adjusted for age, sex, education, and APOE $\varepsilon 4$ genotype status. The sample included $997 \mathrm{CU} / \mathrm{A}-, 446 \mathrm{CU} / \mathrm{A}+, 78 \mathrm{MCl} / \mathrm{A}-$, and $106 \mathrm{MCl} / \mathrm{A}+$ persons. For most NPS, the highest frequency of NPS was found in MCI/A+ and the lowest in CU/A-. The odds ratios of having NPS, depression (BDI $\geq 13$ ), or anxiety (BAI $\geq 8, \geq 10$ ) were consistently highest for $\mathrm{MCl} / \mathrm{A}+$ participants. In conclusion, $M C l$ with $A \beta$ burden of the brain is associated with an increased risk of having NPS as compared to MCI without $A \beta$ burden. This implies that the underlying Alzheimer's disease biology (i.e., cerebral A $\beta$ amyloidosis) may drive both cognitive and psychiatric symptoms.
\end{abstract}

\section{Introduction}

Neuropsychiatric symptoms (NPS) are risk factors for mild cognitive impairment (MCI) and Alzheimer's disease (AD) dementia ${ }^{1-5}$. Furthermore, there is growing evidence of an association between NPS and neuroimaging biomarker abnormality in the context of brain aging.

Although some studies have examined the associations between NPS, particularly depression and anxiety, and

\footnotetext{
Correspondence: Yonas E. Geda (geda.yonas@mayo.edu)

${ }^{1}$ Translational Neuroscience and Aging Laboratory, Mayo Clinic, Scottsdale, AZ, USA

${ }^{2}$ Institute of Sports and Sports Science, Karlsruhe Institute of Technology, Karlsruhe, Germany

Full list of author information is available at the end of the article.
}

cortical amyloid deposition among cognitively normal ${ }^{6-8}$ or impaired participants ${ }^{9-14}$, these studies have been limited by small sample sizes, have not determined whether the associations between NPS and elevated brain amyloid differ by cognitive status (cognitively unimpaired (CU) vs. MCI), or have not conducted subanalyses among persons with amnestic MCI (aMCI). aMCI coupled with amyloid positivity is considered the typical prodromal stage of dementia due to $\mathrm{AD}^{15}$.

We sought to examine the frequency of NPS among community-dwelling non-demented participants (CU; MCI) by brain amyloid status (abnormal, $\mathrm{A}+$ vs. normal, $\mathrm{A}-$ ). We then investigated whether cognitive/amyloid status was associated with the odds of having NPS. We also conducted 
secondary analyses among CU and aMCI participants. Based on previous literature, we hypothesized that persons with MCI and elevated amyloid burden would have higher odds of having NPS as compared to MCI persons without elevated amyloid burden or $\mathrm{CU}$ participants.

\section{Materials and methods \\ Design and sample}

This cross-sectional study was derived from the ongoing, population-based Mayo Clinic Study of Aging (MCSA) in Olmsted County, $\mathrm{MN}^{16}$. Eligible participants were non-demented and $\geq 50$ years of age, with a concurrent, valid NPS assessment and amyloid positron emission tomography (PET) neuroimaging. The institutional review boards of the Mayo Clinic and Olmsted Medical Center in Rochester, MN approved the MCSA protocols. All study participants provided written informed consent.

\section{Neurocognitive evaluation}

Participants underwent a face-to-face evaluation including a neurological examination, a study coordinator visit, and neuropsychological testing. The reader is referred elsewhere for details on the face-to-face evaluation ${ }^{16}$. Briefly, the neurological evaluation comprised a neurological history review, administration of the Short Test of Mental Status ${ }^{17}$, and a neurological examination. The study coordinator visit included the Clinical Dementia Rating Scale $(C D R)^{18}$. Neuropsychological testing was administered by a psychometrist in order to assess performance in four cognitive domains: memory (delayed recall trials from Auditory Verbal Learning Test $^{19}$, Wechsler Memory Scale-Revised ${ }^{20}$, Logical Memory and Visual Reproduction subtests), language (Boston Naming Test ${ }^{21}$, category fluency ${ }^{22}$ ); visuospatial skills (Wechsler Adult Intelligence Scale-Revised ${ }^{23}$, Picture Completion and Block Design subtests); and attention/executive function (Trail-Making Test Part $\mathrm{B}^{24}$, Wechsler Adult Intelligent Scale-Revised ${ }^{23}$, Digit Symbol Substitution subtest). An expert consensus panel consisting of physicians, study coordinators, and neuropsychologists reviewed the results for each participant and determined whether a participant was $\mathrm{CU}$ or had MCI based on published criteria. Individuals were classified as CU based on normative data developed in this community $^{25-28}$. For MCI, the revised Mayo Clinic criteria for $\mathrm{MCI}^{29,30}$ were used: (1) cognitive concern expressed by a physician, informant, participant, or nurse; (2) impairment in one or more cognitive domains (executive functions, memory, language, or visuospatial skills); (3) essentially normal functional activities; and (4) absence of dementia. Participants with MCI had a CDR score of 0 or 0.5 ; however, the final diagnosis of MCI was based on all available data.

\section{Assessment of NPS}

We measured NPS using the Neuropsychiatric Inventory Questionnaire (NPI-Q) ${ }^{31}$. The NPI-Q is designed to assess 12 emotional behaviors (i.e., agitation, delusion, hallucination, depression, anxiety, euphoria, apathy, disinhibition, irritability, aberrant motor behavior, sleep, and eating/appetite) and was administered as a structured interview to an informant by a research nurse or study coordinator. In addition to the NPI-Q, participants completed the Beck Depression Inventory-II (BDI-II) ${ }^{32}$ and Beck Anxiety Inventory (BAI) ${ }^{33}$. Both inventories are validated and consist of 21 items that measure common symptoms of depression (such as feeling guilty or loss of interest) over the past 2 weeks and symptoms of anxiety (such as nervousness or fear of losing control) over the past 1 week. The severity of each symptom is rated on a Likert scale ranging from 0 to 3 , with a total score ranging from 0 to 63 for BDI-II and BAI, respectively.

\section{Molecular imaging: PiB-PET acquisition}

We performed amyloid PET imaging using the Pittsburgh Compound B tracer. The reader is referred elsewhere for details on PiB-PET imaging in the MCSA ${ }^{34,35}$. Briefly, PiB scans, consisting of four 5-min dynamic frames, were acquired from 40 to $60 \mathrm{~min}$ after intravenous injection with $292-728 \mathrm{MBq}$ of ${ }^{11} \mathrm{C}$-PiB. Images were analyzed using an in-house, fully automated image processing pipeline in which image voxel values were extracted from automatically labeled regions of interest propagated from regions defined on each participant's own magnetic resonance imaging (MRI). A global amyloid PET standardized uptake value ratio (SUVR) was formed from the prefrontal, orbitofrontal, parietal, temporal, anterior cingulate, and posterior cingulate/precuneus regions of interest and normalized to the cerebellar gray matter. Participants with an SUVR $>1.42$ were classified as having an abnormal PiB-PET retention (elevated $\beta$ amyloid burden; $\mathrm{A}+$ ) as we have previously validated by autopsy $^{36}$.

\section{Statistical analysis}

In this study, we created four groups based on cognitive/amyloid status: (1) CU with normal amyloid deposition (CU/A-; $n=997) ;(2) \mathrm{CU}$ with elevated brain amyloid deposition (CU/A+; $n=446)$; (3) MCI with normal amyloid deposition (MCI/A-; $n=78)$; and (4) MCI with elevated brain amyloid deposition (MCI/A+; $n=106)$. In a first step, we compared baseline characteristics and frequency of NPS between these four groups, using Kruskal-Wallis and Fisher's Exact tests (for continuous outcomes such as age; reported as median and interquartile range (IQR)) and Chi-square tests (for categorical outcomes such as male sex; reported as number and percentage, \%). We then used multivariable 
logistic regression models to compute odds ratios (OR) and $95 \%$ confidence intervals ( $95 \% \mathrm{CIs}$ ) to compare the odds of having NPS between these four groups. In the regression analyses, we defined CU/A- persons as the reference group. NPS were the presumed dependent variables, and cognitive/amyloid status groups were the presumed independent variables. We ran the regression analyses on depression, anxiety, apathy, irritability, nighttime behavior, any NPS, any non-psychotic NPS (all measured through NPI-Q) and excluded other symptoms measured by NPI-Q (i.e., agitation, motor behavior, appetite/eating change, delusions, hallucinations, euphoria, disinhibition, and any psychotic NPS) due to low number of events. In order to examine the odds of clinically significant depression and anxiety, we also ran the analyses for $\mathrm{BDI}-\mathrm{II} \geq 13, \mathrm{BAI} \geq 8$, and $\mathrm{BAI} \geq 10$. Finally, we conducted secondary analyses comparing $\mathrm{CU}$ (CU/A-; CU/A+) and amnestic MCI participants (aMCI/ $\mathrm{A}-; \mathrm{aMCI} / \mathrm{A}+$ ). All analyses were adjusted for traditional confounders, i.e., age, sex, education, as well as Apolipoprotein (APOE) $\varepsilon 4$ genotype status. Analyses were done using the two-tailed alpha level of 0.05 and performed with SAS 9.4 (SAS Institute, Cary, NC).

\section{Results}

\section{Demographics}

The sample consisted of 1627 persons (54.3\% males). The median (IQR) age was $72.7(64.5,79.3)$ years. Nine hundred and ninety-seven participants were CU/A-, 446 $\mathrm{CU} / \mathrm{A}+, 78 \mathrm{MCI} / \mathrm{A}-$, and 106 were $\mathrm{MCI} / \mathrm{A}+$. Four hundred and fifty-four participants $(28.4 \%)$ were APOE $\varepsilon 4$ carriers (Table 1). The MCI/A - group had a significantly higher percentage of males compared to the other groups.
Participants who were $\mathrm{MCI} / \mathrm{A}+$ had the highest median age, and participants who were CU/A- had the lowest median age. For an overview of demographics for the $\mathrm{CU}$ and aMCI sample, please refer to Supplementary Table 1.

\section{Presence of NPS}

The $\mathrm{MCI} / \mathrm{A}+$ group had the highest frequency of any NPS (59.4\%), followed by MCI/A- (34.6\%), CU/A+ (24.9\%), and CU/A - (20.0\%; Table 2). Psychotic symptoms were rare across groups. The MCI/A+ group had the highest frequency of persons with clinical depression $(B D I-I I \geq 13)$ and clinical anxiety (BAI $\geq 10)$. Similarly, in the secondary analyses including aMCI and $\mathrm{CU}$ participants, the aMCI/A+ group had the highest frequency of any NPS (59.3\%), followed by aMCI/A - (35.7\%), CU/A+ (24.9\%), and CU/A - (20\%). Please refer to Supplementary Table 2 for display of data.

\section{Association between NPS and cognitive/amyloid status}

After adjusting for age, sex, education, and APOE $\varepsilon 4$ carrier status, the odds of having any NPS as measured by NPI-Q (i.e. depression, anxiety, apathy, irritability, nighttime behavior, any NPS, and any non-psychotic NPS), depression (BDI-II $\geq 13)$, or anxiety $(\mathrm{BAI} \geq 8, \mathrm{BAI} \geq 10$ ) were significantly increased for the $\mathrm{MCI} / \mathrm{A}+$ group as compared to the reference group (CU/A-) for all variables (Table 3). MCI/A - participants had significantly increased odds of having anxiety, apathy, irritability, any NPS, any non-psychotic NPS, BAI $\geq 8$, and BAI $\geq 10$ as compared to the reference group. In the secondary analyses including $\mathrm{CU}$ and aMCI participants, there was a similar trend: aMCI/A+ participants, as compared to CU/ A-, had significantly increased odds of having NPS (all

Table 1 Demographic characteristics of study participants

\begin{tabular}{|c|c|c|c|c|c|c|}
\hline & $\mathrm{CU} / \mathrm{A}-(n=997)$ & $\mathrm{CU} / \mathrm{A}+(n=446)$ & $\mathrm{MCl} / \mathrm{A}-(n=78)$ & $\mathrm{MCl} / \mathrm{A}+(n=106)$ & $\begin{array}{l}\text { Total } \\
(n=1627)\end{array}$ & $p$ \\
\hline Male sex, $N(\%)$ & $539(54.1)$ & $232(52.0)$ & $59(75.6)$ & $54(50.9)$ & $884(54.3)$ & $0.001^{\mathrm{a}}$ \\
\hline Age, years & $68.7(61.3,75.8)$ & $77.2(71.5,82.1)$ & $76.7(69.3,82.8)$ & $81.3(75.8,86.0)$ & $72.7(64.5,79.3)$ & $<0.001^{b}$ \\
\hline Age, $N(\%)$; years & & & & & & $<0.001^{a}$ \\
\hline $50-69$ & $545(54.7)$ & $89(20.0)$ & $20(25.6)$ & $6(5.7)$ & $660(40.6)$ & \\
\hline $70-95$ & $452(45.3)$ & 357 (80.0) & $58(74.4)$ & $100(94.3)$ & $967(59.4)$ & \\
\hline Education, years & $15.0(13.0,16.0)$ & $14.0(12.0,16.0)$ & $12.0(12.0,16.0)$ & $13.0(12.0,16.0)$ & $14.0(12.0,16.0)$ & $<0.001^{b}$ \\
\hline$>12$ years, $N(\%)$ & $758(76.0)$ & $316(70.9)$ & $38(48.7)$ & $58(54.7)$ & $1170(71.9)$ & $<0.001^{\mathrm{a}}$ \\
\hline APOE $\varepsilon 4$ carrier, $N(\%)$ & $205(21.0)^{\{19\}}$ & $183(41.5)^{\{5\}}$ & $13(17.1)^{\{2\}}$ & $53(50.5)^{\{1\}}$ & $454(28.4)^{\{27\}}$ & $<0.001^{a}$ \\
\hline
\end{tabular}

Data presented are median (interquartile range) unless otherwise noted. Significant $p$ values appear bold

$C U$ cognitively unimpaired, $M C l$ mild cognitive impairment, $A-$ normal PiB-PET, $A+$ abnormal PiB-PET, APOE $\varepsilon 4$ Apolipoprotein $\varepsilon 4$, ${ }^{\{N\}}$ number of missing data ${ }^{\text {a } C h i-S q u a r e ~ t e s t ~}$

${ }^{\text {b}}$ Kruskal-Wallis test 
Table 2 Frequency of neuropsychiatric symptoms across cognitive/amyloid status groups

\begin{tabular}{|c|c|c|c|c|c|c|}
\hline & CU/A- $(n=997)$ & $\mathrm{CU} / \mathrm{A}+(n=446)$ & $\mathrm{MCl} / \mathrm{A}-(n=78)$ & $\mathrm{MCl} / \mathrm{A}+(n=106)$ & $\begin{array}{l}\text { Total } \\
(n=1627)\end{array}$ & $p$ \\
\hline \multicolumn{7}{|l|}{ Non-psychotic NPS } \\
\hline Agitation & $15(1.5)$ & $12(2.7)$ & $5(6.4)$ & $11(10.4)$ & $43(2.6)$ & $<0.001^{d}$ \\
\hline Depression/dysphoria & $100(10.0)$ & $51(11.4)$ & $10(12.8)$ & $31(29.2)$ & $192(11.8)$ & $<0.001^{\mathrm{e}}$ \\
\hline Anxiety & $42(4.2)$ & $28(6.3)$ & $9(11.5)$ & $21(19.8)$ & $100(6.1)$ & $<0.001^{\mathrm{e}}$ \\
\hline Apathy & $32(3.2)$ & $26(5.8)$ & $7(9.0)$ & $24(22.6)$ & $89(5.5)$ & $<0.001^{\mathrm{e}}$ \\
\hline Irritability & $68(6.8)$ & $40(9.0)$ & $16(20.5)$ & $25(23.6)$ & $149(9.2)$ & $<0.001^{\mathrm{e}}$ \\
\hline Motor behavior & $8(0.8)$ & $3(0.7)$ & $3(3.8)$ & $5(4.7)$ & $19(1.2)$ & $0.002^{d}$ \\
\hline Nighttime behavior ${ }^{a}$ & $40(4.5)$ & $32(7.9)$ & $5(7.0)$ & $17(19.5)$ & $94(6.4)$ & $<0.001^{\mathrm{e}}$ \\
\hline Appetite/eating change & $31(3.1)$ & $14(3.1)$ & $3(3.8)$ & $16(15.1)$ & $64(3.9)$ & $<0.001^{\mathrm{d}}$ \\
\hline \multicolumn{7}{|l|}{ Psychotic NPS } \\
\hline Delusions & $1(0.1)$ & $1(0.2)$ & $2(2.6)$ & $3(2.8)$ & $7(0.4)$ & $<0.001^{d}$ \\
\hline Hallucinations & $0(0.0)$ & $2(0.4)$ & $0(0.0)$ & $2(1.9)$ & $4(0.2)$ & $0.008^{d}$ \\
\hline Euphoria & $4(0.4)$ & $3(0.7)$ & $0(0.0)$ & $1(0.9)$ & $8(0.5)$ & $0.591^{d}$ \\
\hline Disinhibition & $10(1.0)$ & $2(0.4)$ & $3(3.8)$ & $7(6.6)$ & $22(1.4)$ & $<0.001^{\mathrm{d}}$ \\
\hline Number of NPS (0-12) & & & & & & $<0.001^{f}$ \\
\hline Mean (SD) & $0.4(0.9)$ & $0.5(1.0)$ & $0.8(1.5)$ & $1.5(1.9)$ & $0.5(1.1)$ & \\
\hline Median (IQR) & $0.0(0.0,0.0)$ & $0.0(0.0,0.0)$ & $0.0(0.0,1.0)$ & $1.0(0.0,3.0)$ & $0.0(0.0,0.0)$ & \\
\hline Any NPS & $199(20.0)$ & $111(24.9)$ & $27(34.6)$ & $63(59.4)$ & $400(24.6)$ & $<0.001^{\mathrm{e}}$ \\
\hline Any non-psychotic NPS & $199(20.0)$ & $111(24.9)$ & $27(34.6)$ & $63(59.4)$ & $400(24.6)$ & $<0.001^{\mathrm{e}}$ \\
\hline Any psychotic NPS & $12(1.2)$ & $8(1.8)$ & $3(3.8)$ & $10(9.4)$ & $33(2.0)$ & $<0.001^{\mathrm{d}}$ \\
\hline BDI-II total ${ }^{b}$ & & & & & & $<0.001^{f}$ \\
\hline Mean (SD) & $4.1(4.7)$ & $4.6(4.4)$ & $4.9(5.0)$ & $7.5(6.5)$ & $4.5(4.8)$ & \\
\hline Median (IQR) & $3.0(1.0,6.0)$ & $4.0(1.0,7.0)$ & $3.0(1.0,8.0)$ & $7.0(2.0,9.0)$ & $3.0(1.0,7.0)$ & \\
\hline $\mathrm{BDI}-\| \geq 13^{\mathrm{b}}$ & $54(5.4)$ & $28(6.3)$ & $8(10.3)$ & $17(16.0)$ & $107(6.6)$ & $<0.001^{\mathrm{e}}$ \\
\hline BAl total $(0-63)^{c}$ & & & & & & $<0.001^{f}$ \\
\hline Mean (SD) & $2.4(3.7)$ & $2.8(4.3)$ & $3.9(5.2)$ & $4.7(5.5)$ & $2.7(4.1)$ & \\
\hline Median (IQR) & $1.0(0.0,3.0)$ & $1.0(0.0,4.0)$ & $2.0(0.0,6.0)$ & $3.0(1.0,7.0)$ & $1.0(0.0,4.0)$ & \\
\hline $\mathrm{BAI} \geq 8^{\mathrm{C}}$ & $86(8.6)$ & $46(10.3)$ & $15(19.2)$ & $23(21.7)$ & $170(10.5)$ & $<0.001^{\mathrm{e}}$ \\
\hline $\mathrm{BAI} \geq 10^{c}$ & $51(5.1)$ & $31(7.0)$ & $8(10.3)$ & $14(13.2)$ & $104(6.4)$ & $0.004^{e}$ \\
\hline
\end{tabular}

Data presented are $N(\%)$ unless otherwise noted. Significant $p$ values appear bold

$C U$ cognitively unimpaired, $M C I$ mild cognitive impairment, $A-$ normal PiB-PET, $A+$ abnormal PiB-PET, SD standard deviation, $I Q R$ interquartile range, $B D I-I /$ Beck Depression Inventory II, BAl Beck Anxiety Inventory

anformation missing for 168 participants

Information missing for 5 participants

Information missing for 3 participants

${ }^{d}$ Fisher's Exact test

${ }^{\text {e}} \mathrm{Chi}$-Square test

${ }^{f}$ Kruskal-Wallis test

NPI-Q variables), depression (BDI-II $\geq 13$ ), or anxiety (BAI $\geq 8, \mathrm{BAI} \geq 10$ ). For aMCI/A- participants, the odds were significantly increased for irritability, any NPS, any non-psychotic NPS, and BAI $\geq 8$ (Supplementary Table 3).

\section{Discussion}

Here we report that community-dwelling older persons with coexistence of MCI and elevated brain amyloid deposition had significantly higher odds of having NPS as 
Table 3 Odds of having neuropsychiatric symptoms by cognitive/amyloid status groups

\begin{tabular}{|c|c|c|c|c|c|}
\hline $\begin{array}{l}\text { Dependent } \\
\text { variable }\end{array}$ & $N$ & $\begin{array}{l}\text { Independent } \\
\text { variable }\end{array}$ & $N$ & OR $(95 \% \mathrm{Cl})$ & $p$ \\
\hline \multirow[t]{4}{*}{ Depression } & 97 & CU/A- & 978 & 1.00 (reference) & \\
\hline & 51 & $\mathrm{CU} / \mathrm{A}+$ & 441 & $1.13(0.76,1.67)$ & 0.55 \\
\hline & 10 & $\mathrm{MCl} / \mathrm{A}-$ & 76 & $1.33(0.65,2.72)$ & 0.43 \\
\hline & 31 & $\mathrm{MCl} / \mathrm{A}+$ & 105 & $3.48(2.05,5.90)$ & $<0.001$ \\
\hline \multirow[t]{4}{*}{ Anxiety } & 42 & CU/A- & 978 & 1.00 (reference) & \\
\hline & 27 & $\mathrm{CU} / \mathrm{A}+$ & 441 & $1.45(0.84,2.50)$ & 0.18 \\
\hline & 9 & $\mathrm{MCl} / \mathrm{A}-$ & 76 & $2.82(1.27,6.25)$ & 0.011 \\
\hline & 21 & $\mathrm{MCl} / \mathrm{A}+$ & 105 & $5.29(2.73,10.23)$ & $<0.001$ \\
\hline \multirow[t]{4}{*}{ Apathy } & 31 & CU/A- & 978 & 1.00 (reference) & \\
\hline & 26 & $\mathrm{CU} / \mathrm{A}+$ & 441 & $1.62(0.90,2.91)$ & 0.11 \\
\hline & 7 & $\mathrm{MCl} / \mathrm{A}-$ & 76 & $2.51(1.03,6.09)$ & 0.042 \\
\hline & 24 & $\mathrm{MCl} / \mathrm{A}+$ & 105 & $7.06(3.59,13.88)$ & $<0.001$ \\
\hline \multirow[t]{4}{*}{ Irritability } & 68 & CU/A- & 978 & 1.00 (reference) & \\
\hline & 39 & $\mathrm{CU} / \mathrm{A}+$ & 441 & $1.32(0.84,2.08)$ & 0.23 \\
\hline & 16 & $\mathrm{MCl} / \mathrm{A}-$ & 76 & $3.39(1.80,6.38)$ & $<0.001$ \\
\hline & 25 & $\mathrm{MCl} / \mathrm{A}+$ & 105 & $4.21(2.34,7.58)$ & $<0.001$ \\
\hline \multirow{4}{*}{$\begin{array}{l}\text { Nighttime } \\
\text { behavior }\end{array}$} & 39 & $\mathrm{CU} / \mathrm{A}-$ & 879 & 1.00 (reference) & \\
\hline & 31 & $\mathrm{CU} / \mathrm{A}+$ & 400 & $1.31(0.77,2.23)$ & 0.32 \\
\hline & 5 & $\mathrm{MCl} / \mathrm{A}-$ & 69 & $1.13(0.42,3.06)$ & 0.81 \\
\hline & 17 & $\mathrm{MCl} / \mathrm{A}+$ & 87 & $3.06(1.52,6.17)$ & 0.002 \\
\hline \multirow[t]{4}{*}{ Any NPS } & 192 & CU/A- & 978 & 1.00 (reference) & \\
\hline & 110 & $\mathrm{CU} / \mathrm{A}+$ & 441 & $1.18(0.88,1.58)$ & 0.27 \\
\hline & 26 & $\mathrm{MCl} / \mathrm{A}-$ & 76 & $1.77(1.06,2.97)$ & 0.029 \\
\hline & 63 & $\mathrm{MCl} / \mathrm{A}+$ & 105 & $4.84(3.06,7.65)$ & $<0.001$ \\
\hline \multirow{4}{*}{$\begin{array}{l}\text { Any non- } \\
\text { psychotic NPS }\end{array}$} & 192 & CU/A- & 978 & 1.00 (reference) & \\
\hline & 110 & $\mathrm{CU} / \mathrm{A}+$ & 441 & $1.18(0.88,1.58)$ & 0.27 \\
\hline & 26 & $\mathrm{MCl} / \mathrm{A}-$ & 76 & $1.77(1.06,2.97)$ & 0.029 \\
\hline & 63 & $\mathrm{MCl} / \mathrm{A}+$ & 105 & $4.84(3.06,7.65)$ & $<0.001$ \\
\hline \multirow[t]{4}{*}{$B D I-I I \geq 13$} & 53 & CU/A- & 977 & 1.00 (reference) & \\
\hline & 28 & $\mathrm{CU} / \mathrm{A}+$ & 438 & $1.29(0.77,2.17)$ & 0.33 \\
\hline & 8 & $\mathrm{MCl} / \mathrm{A}-$ & 76 & $1.70(0.75,3.87)$ & 0.20 \\
\hline & 17 & $\mathrm{MCl} / \mathrm{A}+$ & 105 & $3.42(1.75,6.71)$ & $<0.001$ \\
\hline \multirow[t]{4}{*}{$B A I \geq 8$} & 83 & CU/A- & 977 & 1.00 (reference) & \\
\hline & 45 & $\mathrm{CU} / \mathrm{A}+$ & 440 & $1.22(0.80,1.86)$ & 0.35 \\
\hline & 15 & $\mathrm{MCl} / \mathrm{A}-$ & 76 & $2.88(1.52,5.44)$ & 0.001 \\
\hline & 23 & $\mathrm{MCl} / \mathrm{A}+$ & 105 & $2.97(1.66,5.30)$ & $<0.001$ \\
\hline
\end{tabular}

Table 3 continued

\begin{tabular}{llllll}
\hline $\begin{array}{l}\text { Dependent } \\
\text { variable }\end{array}$ & $\boldsymbol{N}$ & $\begin{array}{l}\text { Independent } \\
\text { variable }\end{array}$ & $\boldsymbol{N}$ & OR (95\% Cl) & $\boldsymbol{p}$ \\
\hline $\mathrm{BAI} \geq 10$ & 50 & $\mathrm{CU} / \mathrm{A}-$ & 977 & 1.00 (reference) & \\
& 30 & $\mathrm{CU} / \mathrm{A}+$ & 440 & $1.45(0.86,2.43)$ & 0.16 \\
& 8 & $\mathrm{MCl} / \mathrm{A}-$ & 76 & $2.31(1.02,5.26)$ & $\mathbf{0 . 0 4 5}$ \\
& 14 & $\mathrm{MCl} / \mathrm{A}+$ & 105 & $3.01(1.47,6.15)$ & $\mathbf{0 . 0 0 3}$ \\
\hline
\end{tabular}

Significant $p$ values appear bold. Analyses adjusted for age, sex, education, and APOE $\varepsilon 4$ genotype status

$C U$ cognitively unimpaired, $M C l$ mild cognitive impairment, $A-$ normal PiB-PET, $A+$ abnormal PiB-PET, $O R$ odds ratio, $C l$ confidence interval, $p$ value comparing to reference group, BDI-II Beck Depression Inventory II, BAI Beck Anxiety Inventory

compared to $\mathrm{CU}$ persons without elevated brain amyloid deposition. For example, the odds of having apathy were 7.1 times higher in MCI/A+ than CU/A- persons. Similarly, the odds for MCI/A+ as compared to CU/Apersons of having anxiety were 5.3 times, of irritability 4.2 times, and of having depression 3.5 times higher. The same observations hold true for aMCI persons in our secondary analyses, i.e., the odds of having NPS for the $\mathrm{aMCI} / \mathrm{A}+$ group were significantly higher than for the reference group. Furthermore, on average, $\mathrm{MCI} / \mathrm{A}$ - persons had the second highest odds of having NPS, and the odds were significantly increased for anxiety, apathy, irritability, any NPS, any non-psychotic NPS, BAI $\geq 8$, and $\mathrm{BAI} \geq 10$ as compared to the reference group (CU/A-). These results changed slightly when we looked at aMCI/A - vs. CU/A - persons; only the increased odds for irritability, any NPS, any non-psychotic NPS, and BAI $\geq 8$ remained significant. Furthermore, we additionally adjusted the analyses for global cognition ( $z$-score) to see whether this would impact the findings. Indeed, we observed that the odds for $\mathrm{MCI} / \mathrm{A}+$ participants were still significantly increased for depression, anxiety, apathy, irritability, any NPS, any non-psychotic NPS, and BDIII $\geq 13$ but no longer remained significantly increased for nighttime behavior, $\mathrm{BAI} \geq 8$, and $\mathrm{BAI} \geq 10$ (data not shown). Similarly, the odds were still significantly increased for $\mathrm{MCI} / \mathrm{A}$ - for irritability and $\mathrm{BAI} \geq 8$ but no longer for anxiety, apathy, any NPS, any non-psychotic NPS, and BAI $\geq 10$ (data not shown).

Overall, our results were in line with our hypothesis that the combined presence of cognitive impairment and elevated brain amyloid deposition is associated with greater odds of having NPS as compared to MCI without amyloid deposition. This indicates that the underlying AD biology as measured by PET A $\beta$ imaging contributes to an increased frequency of NPS over and above expected from MCI status alone, which implies that the underlying $\mathrm{AD}$ biology may drive both the cognitive symptoms and 
psychiatric symptoms. Of note, 78 of our participants were $\mathrm{MCI} / \mathrm{A}-$. The widely held interpretation is that the underlying neurobiological substrate of an amyloid negative $\mathrm{MCI}$ is most likely to be non-AD; whereas MCI with abnormal amyloid is interpreted as $\mathrm{MCI}$ due to $\mathrm{AD}^{37}$. In addition, even though the $\mathrm{MCI} / \mathrm{A}+$ group was about 10 years older than the CU/A- group, our findings are over and above what can be explained by age since we have rigorously adjusted for age.

Our findings should also be interpreted within the context of published literature. We and others have previously reported cross-sectional associations between NPS, particularly depression, anxiety, and apathy, with increased amyloid deposition in cognitively normal ${ }^{6,8}$ or cognitively impaired persons ${ }^{10,11}$, whereas few studies found no association ${ }^{9}$ or only in subgroup analyses ${ }^{7}$. To our knowledge, there is only a limited number of crosssectional studies that examined the association between NPS and cortical amyloid in both cognitively normal and cognitively impaired persons. French researchers reported that, in a sample comprised of CU persons as well as MCI and $\mathrm{AD}$ patients, anxiety and irritability were associated with higher cortical amyloid deposition in various regions of interest; the number of significant correlations was reduced in subgroup analyses among $\mathrm{MCI}$ or $\mathrm{AD}$ only ${ }^{12}$. Similarly, researchers from UCLA observed that the relationship between depression and anxiety with amyloid deposition varied between cognitively normal and MCI persons, i.e., depression and anxiety in MCI were associated with amyloid deposition in different regions of interest than in cognitively normal persons ${ }^{13}$. Finally, a group of investigators from Pittsburgh found that $\mathrm{PiB}$ retention in three out of six depressed MCI patients was comparable to $\mathrm{PiB}$ retention in $\mathrm{AD}$ patients ${ }^{14}$. However, these studies differ from ours in various ways, i.e., our study had a larger, population-based sample, and we wanted to know whether combined presence of cognitive impairment and abnormal amyloid deposition was associated with the odds of having NPS. Our study is also in line with a recently published longitudinal study from Harvard Medical School reporting that higher amyloid beta burden was associated with increasing anxiousdepressive symptoms over time in cognitively normal older individuals ${ }^{38}$. Our current study may also be relevant to the ISTAART-AA criteria for mild behavioral impairment (MBI) and the recently published MBI checklist $(\mathrm{MBI}-\mathrm{C})^{39}$. The construct of MBI refers to a change in behavior or personality in persons without dementia aged $\geq 50$ years that may be the case in a majority of our study population.

The strengths of our study are the large-scale, population-based sample and the rigorous acquisition of
NPS using three validated assessments that are widely used in aging research. The limitations pertain to the relatively small number of participants reporting NPS. However, this was expected given that participants are community-dwelling individuals aged $\geq 50$ years and similar frequencies of NPS in community-based samples have been reported by our group and others in the past $^{40,41}$. Owing to the low number, we were not able to conduct regression analyses stratified by APOE $\varepsilon 4$ status, but we adjusted our analyses for this potential confounder. Furthermore, in order to avoid an assumption violation of the logistic regression, we did not run the analyses on all NPS that are listed in Table 2 and Supplementary Table 2, i.e., we excluded agitation, motor behavior, appetite change, delusions, hallucinations, euphoria, disinhibition, and any psychotic NPS due to low number of events. Another limitation is that we did not take into account the severity of NPS as assessed by the NPI-Q and instead only examined the presence vs. absence of symptoms. However, we included clinical depression and anxiety as measured by BDI-II (score $\geq 13$ ) and BAI (score $\geq 8$; score $\geq 10$ ) in our analyses, even though the scales do not take into consideration whether the symptoms are acute or chronic. For example, a person who has recurrent depression and is successfully treated with antidepressant medication may be symptomfree within 2 weeks of completing the BDI-II questionnaire. We also did not include or adjust the analyses for other neuroimaging biomarkers such as structural MRI of hippocampal volumes and frontal/global cortical atrophy, and we did not investigate various regions of interest but rather a global amyloid PET SUVR. In addition, we did not adjust our analyses for multiple comparisons. However, when considering a Bonferroni correction for Table 3, the alpha significance level would be 0.00167 (i.e., $0.05 / 30$ since we have a total of 10 models with 3 comparisons per model). Thus 10 out of the 17 significant $p$ values still remain significant and none of our major conclusions are affected by the correction. Finally, given the cross-sectional nature of our study, the direction of causality between NPS with amyloid and cognitive status cannot be determined. The current study did not investigate underlying mechanisms that may explain the association between cognitive/amyloid status with NPS. We and others have previously proposed potential mechanisms linking NPS with cognitive outcomes and neuroimaging biomarkers, such as etiologic pathway, shared risk factor or confounding, reverse causality, and/or interaction ${ }^{42,43}$.

Our cross-sectional data show that community-dwelling persons with combined presence of $\mathrm{MCI}$ and amyloid positivity as measured by PiB-PET are at significantly 
increased risk of having NPS. These findings add valuable information on the association between NPS and cognitive/amyloid status. Furthermore, they underline the importance of a thorough assessment as well as potential treatment of NPS in the clinical practice, particularly among persons with confirmed neuroimaging biomarker abnormality who are at risk for AD. The findings need to be confirmed by a prospective cohort study.

\section{Acknowledgements}

Support for this research was provided by $\mathrm{NIH}$ grants: National Institute on Aging (R01 AG057708; U01 AG006786; P50 AG016574; K01 AG028573; R01 AG034676; R01 AG041851; R01 AG011378) and National Institute of Mental Health (K01 MH068351). This project was also supported by F. Hoffman-La Roche, the Robert Wood Johnson Foundation, the Robert H. and Clarice Smith and Abigail Van Buren Alzheimer's Disease Research Program, the GHR Foundation, the Mayo Foundation for Medical Education and Research, Project LQ1605 from the National Program of Sustainability II (MEYS CR), the Edli Foundation, and the Arizona Alzheimer's Consortium

\section{Author details}

${ }^{1}$ Translational Neuroscience and Aging Laboratory, Mayo Clinic, Scottsdale, AZ, USA. ${ }^{2}$ Institute of Sports and Sports Science, Karlsruhe Institute of Technology, Karlsruhe, Germany. ${ }^{3}$ Department of Health Sciences Research, Mayo Clinic, Rochester, MN, USA. ${ }^{4}$ Department of Neurology, Mayo Clinic, Rochester, MN, USA. ${ }^{5}$ Department of Radiology, Mayo Clinic, Rochester, MN, USA. ${ }^{6}$ Department of Psychiatry and Psychology, Mayo Clinic, Rochester, MN, USA. ${ }^{7}$ International Clinical Research Center/St. Anne Hospital, Brno, Czech Republic. ${ }^{8} \mathrm{~F}$. HoffmannLa Roche Ltd, Basel, Switzerland. ${ }^{9}$ Department of Psychiatry and Psychology, Mayo Clinic, Scottsdale, AZ, USA. ${ }^{10}$ Department of Neurology, Mayo Clinic, Scottsdale, AZ, USA

\section{Conflict of interest}

J.K.-R. receives funding from $\mathrm{NIH}$ and Roche. M.V. receives research funding from NIH, Roche, and Biogen. M.M.M. has consulted for Eli Lilly and Lysosomal Therapeutics, Inc. and receives unrestricted research grants from Biogen and Lundbeck, and research funding from the NIH/NIA and Department of Defense. W.K.K. receives research funding from Department of Defense, NIH, Astra Zeneca, Biogen, and Roche. V.J.L. serves on scientific advisory boards for Bayer Schering Pharma, Piramal Life Sciences, and Merck Research and receives research support from GE Healthcare, Siemens Molecular Imaging, AVID Radiopharmaceuticals, and the NIH (NIA, NCI). P.V. receives NIH funding. M.M. M. receives funding from NIH. L.M.B. and M.T. are full-time employees of, and own shares at, F. Hoffmann-La Roche Ltd. C.R.J. serves on scientific advisory board for Eli Lilly and IDSMB for Roche. But he receives no compensation from any commercial entity. He receives research support from the NIH/NIA and the Alexander Family Alzheimer's Disease Research Professorship of the Mayo Foundation. D.S.K. serves on a Data Safety Monitoring Board for the DIAN study. He is an investigator in clinical trials sponsored by Lilly Pharmaceuticals, Biogen, and the Alzheimer's Treatment and Research Institute at USC and receives research support from the NIH. R.O.R. receives research funding from the NIH/NIA, Biogen, and Roche. R.C.P. is a consultant for Roche, Biogen, Merck, Eli Lilly, and Genentech. He receives publishing royalties from Mild Cognitive Impairment (Oxford University Press, 2003) and research support from the NIH. Y.E.G. receives funding from $\mathrm{NIH}$ and Roche and serves on Lundbeck Advisory Board. The other authors declare that they have no conflict of interest.

\section{Publisher's note}

Springer Nature remains neutral with regard to jurisdictional claims in published maps and institutional affiliations.

Supplementary information accompanies this paper at (https://doi.org/ 10.1038/s41398-019-0456-z).

Received: 11 February 2019 Accepted: 16 February 2019

Published online: 28 March 2019

\section{References}

1. Geda, Y. E. et al. Baseline neuropsychiatric symptoms and the risk of incident mild cognitive impairment: a population-based study. Am. J. Psychiatry 171 572-581 (2014).

2. Pink, A. et al. Neuropsychiatric symptoms, APOE epsilon4, and the risk of incident dementia: a population-based study. Neurology 84, 935-943 (2015).

3. Teng, E., Lu, P. H. \& Cummings, J. L. Neuropsychiatric symptoms are associated with progression from mild cognitive impairment to Alzheimer's disease. Dement. Geriatr. Cogn. Disord. 24, 253-259 (2007).

4. Brodaty, $H$. et al. Neuropsychiatric symptoms in older people with and without cognitive impairment. J. Alzheimers Dis. 31, 411-420 (2012).

5. Rosenberg, P. B. et al. The association of neuropsychiatric symptoms in $\mathrm{MCl}$ with incident dementia and Alzheimer disease. Am. J. Geriatr. Psychiatry 21, 685-695 (2013).

6. Krell-Roesch, J. et al. Depressive and anxiety symptoms and cortical amyloid deposition among cognitively normal elderly persons: the Mayo Clinic Study of Aging. Int. Psychogeriatr. 30, 245-251 (2018).

7. Holmes, S. E. et al. beta-Amyloid, APOE and BDNF genotype, and depressive and anxiety symptoms in cognitively normal older women and men. Am. J. Geriatr. Psychiatry 24, 1191-1195 (2016).

8. Yasuno, F. et al. High amyloid-beta deposition related to depressive symptoms in older individuals with normal cognition: a pilot study. Int. J. Geriatr. Psychiatry 31, 920-928 (2016).

9. Chung, J. K. et al. Cortical amyloid beta deposition and current depressive symptoms in alzheimer disease and mild cognitive impairment. J. Geriatr. Psychiatry Neurol. 29, 149-159 (2016).

10. Mori, T. et al. Apathy correlates with prefrontal amyloid beta deposition in Alzheimer's disease. J. Neurol. Neurosurg. Psychiatry 85, 449-455 (2014).

11. Marshall, G. A. et al. Apathy is associated with increased amyloid burden in mild cognitive impairment. J. Neuropsychiatry Clin. Neurosci. 25, 302-307 (2013).

12. Bensamoun, D. et al. Associations between neuropsychiatric symptoms and cerebral amyloid deposition in cognitively impaired elderly people. J. Alzheimers Dis. 49, 387-398 (2016).

13. Lavretsky, $H$. et al. Depression and anxiety symptoms are associated with cerebral FDDNP-PET binding in middle-aged and older nondemented adults. Am. J. Geriatr. Psychiatry 17, 493-502 (2009).

14. Butters, M. A. et al. Imaging Alzheimer pathology in late-life depression with PET and Pittsburgh Compound-B. Alzheimer Dis. Assoc. Disord. 22, 261-268 (2008).

15. Petersen, R. C. Mild cognitive impairment. Contin. (Minneap. Minn.) 22 (2Dementia), 404-418 (2016).

16. Roberts, R. O. et al. The Mayo Clinic Study of Aging: design and sampling participation, baseline measures and sample characteristics. Neuroepidemiology 30, 58-69 (2008).

17. Kokmen, E., Smith, G. E., Petersen, R. C., Tangalos, E. \& Ivnik, R. C. The short test of mental status: correlations with standardized psychometric testing. Arch. Neurol. 48, 725-728 (1991).

18. Morris, J. C. The Clinical Dementia Rating (CDR): current version and scoring rules. Neurology 43, 2412-2414 (1993).

19. Rey, A. L'examen Clinique en Psychologie (Presses Universitaires de France, Paris, 1964).

20. Wechsler, D. Wechsler Memory Scale-Revised (The Psychological Corporation, New York, 1987).

21. Kaplan, E., Goodglass, H. \& Weintraub, S. Boston Naming Test 2nd edn (Lippincott Williams \& Wilkins, Philadelphia, 2001).

22. Lucas, J. A. et al. Mayo's Older Americans Normative Studies: category fluency norms. J. Clin. Exp. Neuropsychol. 20, 194-200 (1998).

23. Wechsler, D. Wechsler Adult Intelligence Scale-Revised (Psychological Corporation, New York, 1981).

24. Reitan, R. M. Validity of the Trail Making Test as an indicator of organic brain damage. Percept. Mot. Skills 8, 271-276 (1958).

25. Ivnik, R. J. et al. Mayo's Older Americans Normative Studies: WAIS-R norms for ages 56 to 97. Clin. Neuropsychol. 6(sup001), 1-30 (1992).

26. Ivnik, R. J. et al. Mayo's Older Americans Normative Studies: WMS-R norms for ages 56 to 94. Clin. Neuropsychol. 6(sup001), 49-82 (1992).

27. Ivnik, R. J. et al. Mayo's Older Americans Normative Studies: updated AVLT norms for ages 56 to 97. Clin. Neuropsychol. 6(sup001), 83-104 (1992).

28. Malec, J. F. et al. Mayo's Older Americans Normative Studies: utility of corrections for age and education for the WAIS-R. Clin. Neuropsychol. 6(sup001) 31-47 (1992). 
29. Petersen, R. C. Mild cognitive impairment as a diagnostic entity. J. Intern. Med. 256, 183-194 (2004)

30. Winblad, B. et al. Mild cognitive impairment-beyond controversies, towards a consensus: report of the International Working Group on Mild Cognitive Impairment. J. Intern. Med. 256, 240-246 (2004).

31. Kaufer, D. I. et al. Validation of the NPI-Q, a brief clinical form of the Neuropsychiatric Inventory. J. Neuropsychiatry Clin. Neurosci. 12, 233-239 (2000).

32. Beck, A. T., Steer, R. A. \& Brown, G. K. BDI-II, Beck Depression Inventory: Manual 2nd edn (Psychological Corp.; Harcourt Brace, San Antonio, TX; Boston, MA, 1996).

33. Beck, A. T. \& Steer, R. A. BAl, Beck Anxiety Inventory: Manual (Psychological Corp., Harcourt Brace Jovanovich, San Antonio, TX, 1990).

34. Jack, C. R. Jr. et al. Defining imaging biomarker cut points for brain aging and Alzheimer's disease. Alzheimers Dement. 13, 205-216 (2017).

35. Lowe, V. J. et al. Comparison of 18 F-FDG and PiB PET in cognitive impairment. J. Nucl. Med. 50, 878-886 (2009).

36. Murray, M. E. et al. Clinicopathologic and 11C-Pittsburgh compound B implications of Thal amyloid phase across the Alzheimer's disease spectrum. Brain 138(Pt 5), 1370-1381 (2015).

37. Albert, M. S. et al. The diagnosis of mild cognitive impairment due to Alzheimer's disease: recommendations from the National Institute on Aging-
Alzheimer's Association workgroups on diagnostic guidelines for Alzheimer's disease. Alzheimers Dement. 7, 270-279 (2011).

38. Donovan, N. J. et al. Longitudinal association of amyloid beta and anxiousdepressive symptoms in cognitively normal older adults. Am. J. Psychiatry 175, 530-537 (2018).

39. Ismail, Z. et al. The Mild Behavioral Impairment Checklist (MBI-C): a rating scale for neuropsychiatric symptoms in pre-dementia populations. J. Alzheimers Dis. 56, 929-938 (2017).

40. Geda, Y. E. et al. Prevalence of neuropsychiatric symptoms in mild cognitive impairment and normal cognitive aging: population-based study. Arch. Gen. Psychiatry 65, 1193-1198 (2008).

41. Lyketsos, C. G. et al. Prevalence of neuropsychiatric symptoms in dementia and mild cognitive impairment: results from the Cardiovascular Health Study. JAMA 288, 1475-1483 (2002).

42. Geda, Y. E., Krell-Roesch, J., Sambuchi, N. \& Michel, B. F. Neuropsychiatric symptoms and neuroimaging biomarkers in alzheimer disease: "which is the cart and which is the horse?". Am. J. Geriatr. Psychiatry 25, 694-696 (2017).

43. Geda, Y. E. et al. Neuropsychiatric symptoms in Alzheimer's disease: past progress and anticipation of the future. Alzheimers Dement. 9, 602-608 (2013). 